\title{
Separation and enrichment of enantiopure from racemic compounds using magnetic levitation
}

\section{Citation}

Yang, Xiaochuan, Shin Yee Wong, David K. Bwambok, Manza B. J. Atkinson, Xi Zhang, George M. Whitesides, and Allan S. Myerson. 2014. "Separation and Enrichment of Enantiopure from Racemic Compounds Using Magnetic Levitation." Chemical Communications. doi:10.1039/ c4cc02604g.

\section{Published Version}

doi:10.1039/c4cc02604g

\section{Permanent link}

http://nrs.harvard.edu/urn-3:HUL.InstRepos:34730475

\section{Terms of Use}

This article was downloaded from Harvard University's DASH repository, and is made available under the terms and conditions applicable to Other Posted Material, as set forth at http:// nrs.harvard.edu/urn-3:HUL.InstRepos:dash.current.terms-of-use\#LAA

\section{Share Your Story}

The Harvard community has made this article openly available.

Please share how this access benefits you. Submit a story.

\section{Accessibility}




\title{
COMMUNICATION
}

Cite this: DOI: 10.1039/x0xx00000x

\section{Separation and Enrichment of Enantiopure from Racemic Compounds Using Magnetic Levitation}

Received 00th January 2012,

Accepted 00th January 2012

\author{
X. Yang ${ }^{a}$, S. Y. Wong ${ }^{a}$, D. K. Bwambok ${ }^{b}$, M. B. J. Atkinson ${ }^{b}$, X. Zhang ${ }^{a}$, G. M. \\ Whitesides $^{b, *}$, A. S. Myerson ${ }^{a, *}$
}

DOI: $10.1039 / \times 0 \times x 00000 x$

www.rsc.org/

Crystallization of a solution with high enantiomeric excess can generate a mixture of crystals of the desired enantiomer and the racemic compound. Using a mixture of S-/RSibuprofen crystals as a model, we demonstrated that magnetic levitation (MagLev) is a useful technique for analysis, separation and enantioenrichment of chiral/racemic products.

The enantiomeric purity of pharmaceutical compounds is important because enantiomers/racemates of a drug can have different effects (i.e. therapeutic or harmful). ${ }^{1}$ In the late $1950 \mathrm{~s}$, the racemic mixture of Thalidomide, for example, was widely prescribed to relieve the symptoms of morning sickness in pregnant women. Nevertheless, the drug was withdrawn in 1962 after a birth defects crisis, where it was later discovered that the (R) enantiomer is effective against morning sickness while the (S) enantiomer causes birth defects. ${ }^{2}$ Apart from therapeutic effects and safety, efficacy of a pure enantiomer is another area of interest. ${ }^{3}$ For example, S-ibuprofen has anti-inflammatory activity that is 150 times higher than that of Ribuprofen. ${ }^{4}$ As a result, this subject is not only of interest to pharmaceutical companies but also to the Food and Drug Administration (FDA) who tends to verifying and marketing most chiral drugs as single enantiomers. In fact, in 2006, approximately $80 \%$ of small-molecule drugs approved by the FDA were chiral and $75 \%$ of them were single enantiomers. ${ }^{5}$ There is need to develop new approaches to analyze enantiomeric purity and obtain pure enantiomers. Asymmetric synthesis, chiral resolution and isolation of enantiomerically pure compounds (e.g., from biological sources) are common methods to produce enantiopure compounds. ${ }^{6}$ In many cases, even with efficient purifications, the products are a mixture of the desired enantiomer and a small or considerable amount of the undesired enantiomer. For example, the active pharmaceutical ingredient used in the treatment of Parkinson's disease (L-3,4dihydroxyphenylalanine), is manufactured with an asymmetric synthesis step yielding 95\%ee. ${ }^{6}$ After synthesis, L-enantiomer is purified by either high-performance liquid chromatography (HPLC) or formation of diastereomers followed by crystallization. These two methods, though commonly used to obtain single enantiomers, have three disadvantages: (i) Separation by HPLC require expensive instrumentation and stationary phase. ${ }^{1}$ (ii) A particular chiral stationary phase may only work for a limited number of compounds. $^{6,7}$ (iii) Formation of diastereomers introduces another compound into the system, and requires additional crystallization and filtration steps. 7

One of the alternative approaches to solution-based separation of enantiomers is to purify the desired enantiomer from a mixture of crystals of enantiomerically pure and racemic compound. Given a solution with high enantiomeric excess, one crystallization step usually generates crystals of the desired enantiomer contaminated with crystals of the racemic compound. ${ }^{8,9}$ Since the racemic compound and pure enantiomers have different densities, MagLev can be used as a cost-effective, and non-destructive method to separate the enantiomerically pure crystals (desired products) from the racemic compound crystals ("impurity").

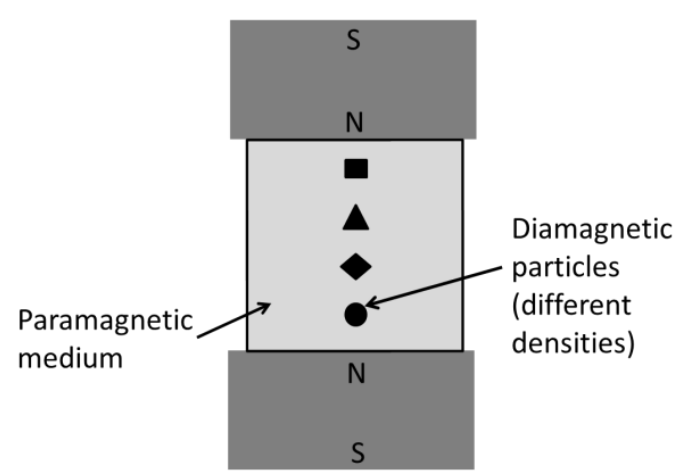

Figure 1. Diamagnetic particles with different densities suspended in a paramagnetic medium can be levitated to different heights when container is placed between the magnets of MagLev device.

The theory of MagLev is described in details elsewhere. ${ }^{10,11,12}$ Briefly, under the influence of an external magnetic field, diamagnetic materials of different densities (suspended in a paramagnetic medium) can be levitated to different heights by the balance of magnetic and gravitational forces (Figure 1). Since most materials (especially most organic compounds) are diamagnetic, 
MagLev can find broad applications in density-based analysis and separations in the pharmaceutical industry. We have demonstrated the use of MagLev to separate mixtures of crystal polymorphs. ${ }^{13}$ Here, we describe the use of MagLev for analysis of enantiomeric purity and enrichment (Figure 2). We selected a mixture of enantiomerically pure and racemic crystals of ibuprofen to demonstrate the concept; S-ibuprofen is metabolically "cleaner" than racemic ibuprofen. ${ }^{14}$ We also performed the separation of Sphebox from RS-phebox (See Supporting Information). Phebox is generally used as an efficient auxiliary for asymmetric catalysis and its enantiomeric purity is vital since it provides appropriate stereochemical and electronic circumstances. To our knowledge, this is the first report on analysis of enantiomer purity, and enantioenrichment based on density using MagLev.

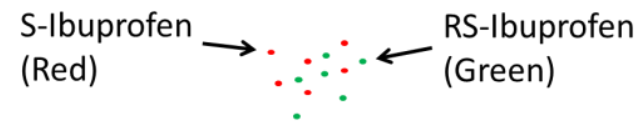

Mixture of enantiopure

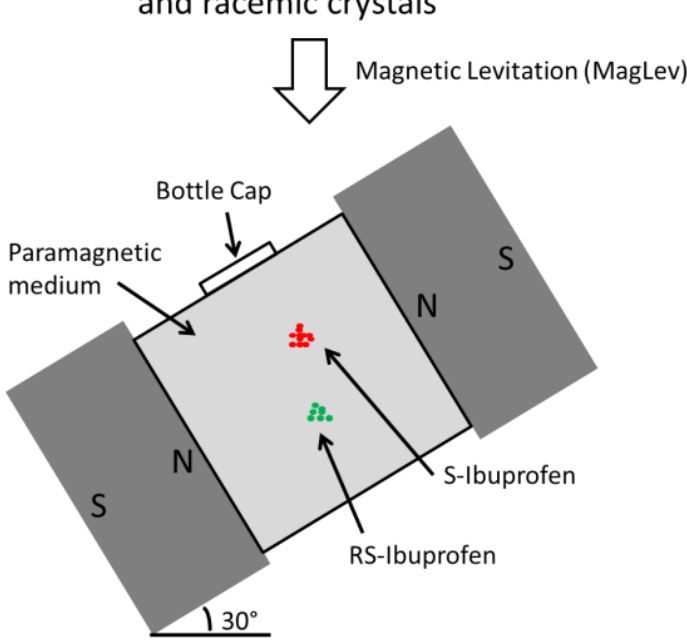

Separation by density
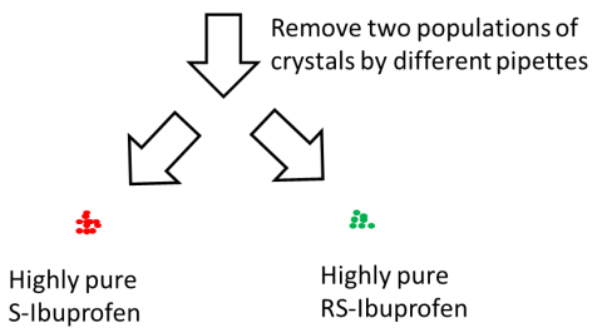

Isolation and analysis<smiles>C1CCCCC1</smiles>

\section{For clinical study or further seeding crystallization}<smiles>C1CCCCC1</smiles>

Recycle for chiral resolution
Figure 2. Schematic illustration of the separation of enantiopure crystals from racemic crystals of ibuprofen by density using MagLev. . Using ibuprofen as a model ystem, MagLev enables separation and isolation of S-lbuprofen and RSlbuprofen by their difference in density. This method can be used as a general method to analyze enantiomeric purity of a mixture or purify enantiomer product for further use. The densities of S-ibuprofen and RS-ibuprofen are reported to be $1.093 \mathrm{~g} / \mathrm{cm}^{3}$ and $1.110 \mathrm{~g} / \mathrm{cm}^{3}$, respectively. ${ }^{15}$
The configuration of the MagLev device that we used is shown in Figure 2. When an object is placed in the container within the MagLev device, the "levitation height" is defined as the distance from the center of the object to the bottom magnet (aligned along the perpendicular distance between two magnets). With this setup, a low-density object will levitate at a higher height compared to a denser object. We separated the enantiomer and racemic crystals of ibuprofen by levitating in aqueous $0.55 \mathrm{M} \mathrm{MnCl}_{2}$ and tilting the MagLev device at $30^{\circ}$ for optimum resolution (see Supporting Information for details).

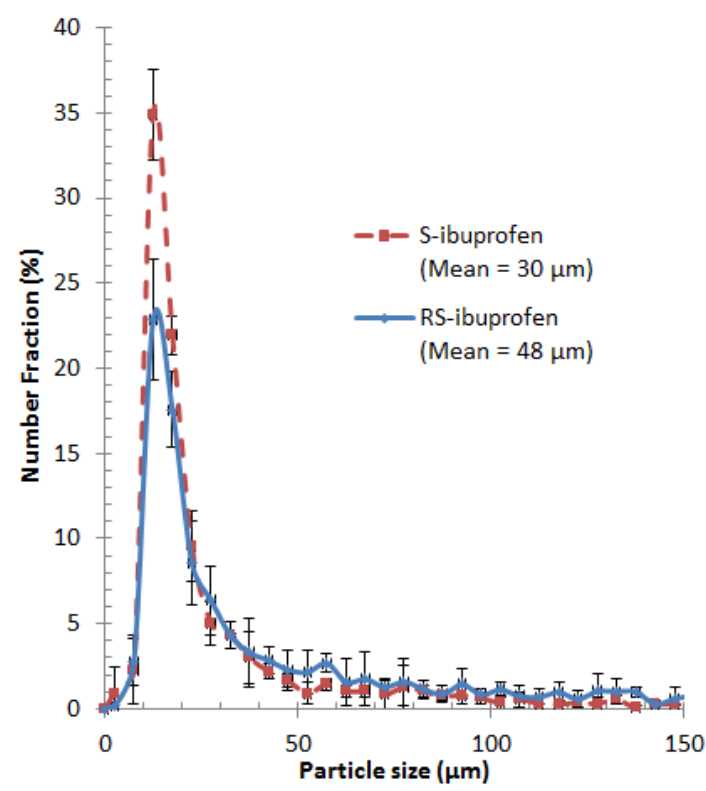

Figure 3. Particle size distribution of S-ibuprofen and RS-ibuprofen crystals measured using optical microscopy. The error bars represent standard deviation from seven independent measurements $(n=7)$.

Particle size distribution is important when performing MagLev because the separation is slower as the particle size decreases (due to both Brownian motion and fluid shear). ${ }^{7}$ Therefore, we performed particle size measurements on ibuprofen crystals using an optical microscope. Figure 3 show that particles of S-ibuprofen (with a mean size of $30 \mu \mathrm{m}$ ) have smaller sizes than those of RS-ibuprofen (with a mean size of $48 \mu \mathrm{m}$ ). The size of these particles is very close to the particle size of the API commonly used in pharmaceutical crystallization processes.

To evaluate the accuracy of MagLev as an analytical tool for measurement of enantiomeric purity, we conducted the separation from prepared ibuprofen mixtures of known compositions. Taking run No. 6 (refer to Table 1) as an example, a prepared mixture of $197.62 \mathrm{mg} \mathrm{S}$ - and $2.61 \mathrm{mg}$ RS- ibuprofen (98.7\% ee) was first dispersed in the glass bottle filled with paramagnetic medium $(0.55 \mathrm{M}$ aqueous manganese chloride). Then the glass bottle was placed in the MagLev device. The Maglev device was tilted at a $30^{\circ}$ in order to increase the separation. ${ }^{7}$ After allowing the mixture to separate overnight ( 12 hours), two populations of crystals were clearly visible in the sample bottle, as shown in Figure 4. The top and the bottom populations levitated to a height of $\sim 19 \mathrm{~mm}$ and $\sim$ $16 \mathrm{~mm}$, respectively. Their separation distance is $\sim 12 \mathrm{~mm}$ (distance between two centers of the populations). The crystals in the top population were carefully removed using a $20 \mathrm{~mL}$ syringe attached to a needle while crystals in the bottom population were collected directly by vacuum filtration. After drying, the mass of the top and 
bottom populations were measured. The crystals in the top population weighed $197.51 \mathrm{mg}$, while the bottom population had $2.33 \mathrm{mg}$ of the crystals. Compared to the total initial mass of 200.23 $\mathrm{mg}$, there was a loss of $0.39 \mathrm{mg}$ during the process (which is probably due to crystals sticking to the glassware or filter paper). During density-separation in MagLev, lower-density objects levitate at higher heights compared to denser objects, thus the top population of crystals should be S-ibuprofen $\left(1.093 \mathrm{~g} / \mathrm{cm}^{3}\right)$ and the bottom population of crystals should be RS-ibuprofen $\left(1.110 \mathrm{~g} / \mathrm{cm}^{3}\right)$. We performed differential scanning calorimetry (DSC) analysis on the crystals to confirm their compositions. Since S-ibuprofen and RSibuprofen have different melting points, the melting peak area was used to quantify composition (see Supporting Information for details).

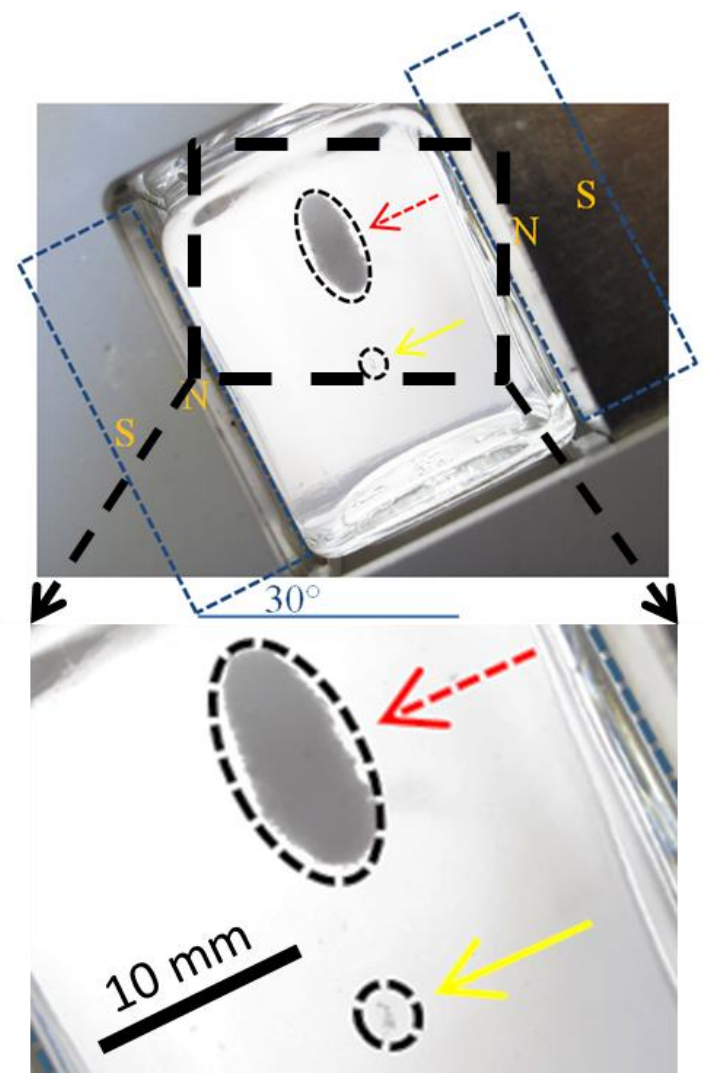

Figure 4. Separation of a solid mixture of $98.7 \%$ S-ibuprofen and $1.3 \% \mathrm{RS}$ ibuprofen in the MagLev device (tilted at $30^{\circ}$ ): Two populations of solid crystals were obtained, S-ibuprofen in the upper portion (red dotted arrow) and RSibuprofen in the bottom portion (yellow arrow). (An enlarged version of the whole photograph is included in supporting information).

Table 1. Enantiomeric purity of S-ibuprofen before and after density-separation from a mixture containing the racemate using MagLev.

\begin{tabular}{c|ccc}
\hline $\begin{array}{c}\text { Run } \\
\text { No. }\end{array}$ & $\begin{array}{c}\text { Mixture (before } \\
\text { separation) }(\% \text { ee })\end{array}$ & $\begin{array}{c}\text { S-ibuprofen } \\
(\% \text { ee) }\end{array}$ & $\begin{array}{c}\text { RS-ibuprofen } \\
(\% \text { ee })\end{array}$ \\
\hline 1 & 48.5 & 95.2 & 5.18 \\
2 & 50.3 & 95.4 & 4.17 \\
3 & 51.0 & 94.9 & 4.26 \\
4 & 79.9 & 98.0 & 3.60 \\
5 & 91.6 & 98.9 & 2.95 \\
6 & 98.7 & 99.8 & 2.57 \\
\hline
\end{tabular}

Both populations of crystals were not $100 \%$ pure. In Run 6 , the top population (197.51 mg) of crystals showed S-ibuprofen with
99.8\% ee purity, indicating that $0.2 \%$ wt of the crystals were RSibuprofen (Table 1). Crystals found at the bottom population (2.33 $\mathrm{mg}$ ) exhibited $2.57 \%$ ee purity, indicating $2.57 \%$ wt of the crystals were S-ibuprofen. Table 1 summarizes the results of six different separations from different initial mixtures. The contaminations in both populations were present in all six separations (and will be discussed in a later section). From Table 1, we see that the reproducibility and selectivity of the separations are good. Run 1-3, starting at similar compositions ( 50\%ee), showed similar separation results: $\sim 95 \%$ ee in the top population with a standard deviation of $\pm 0.2 \%$. In addition, Run 6 with an initial purity of $98.7 \%$ ee could be separated into two distinguishable populations.

Table 2. Comparison of enantiomeric purity analyzed using MagLev to the actual values calculated from the composition of the prepared mixture.

\begin{tabular}{c|ccc}
\hline $\begin{array}{c}\text { Run } \\
\text { No. }\end{array}$ & $\begin{array}{c}\text { Esimated value by } \\
\text { MagLev }(\% \text { ee })\end{array}$ & $\begin{array}{c}\text { Actual value } \\
(\% \text { ee })\end{array}$ & Error(\% ee) \\
\hline 1 & 48.1 & 48.5 & -0.4 \\
2 & 50.6 & 50.3 & +0.3 \\
3 & 51.5 & 51.0 & +0.5 \\
4 & 80.5 & 79.9 & +0.6 \\
5 & 92.3 & 91.6 & +0.7 \\
6 & 98.8 & 98.7 & +0.1 \\
\hline
\end{tabular}

We determined enantiomeric purity using integrated melting peak areas from DSC analysis (See Supporting Information for details). For convenience, we define racemic crystals in a bulk of enantiopure crystals or enantiopure crystals in a bulk of racemic crystals as "impurity" crystals. The analysis showed enantioenrichment although traces of the "impurity" crystals exist in each population. This is probably due to the fact that the "impurity" crystals in both populations are present in small amounts $(<\sim 5 \% \mathrm{wt})$, and thus do not show significant effect on the analysis. Table 2 summarizes the results of enantiomeric purity analysis using MagLev. Taking run 6 as an example, the top and bottom populations weighed $197.51 \mathrm{mg}$ and $2.33 \mathrm{mg}$. Assuming that $197.51 \mathrm{mg}$ are all S-ibuprofen crystals and $2.33 \mathrm{mg}$ are all RS-ibuprofen, the enantiomeric purity of the initial mixture is calculated to be $98.8 \%$ ee (estimated value by MagLev). The mixture was prepared with $197.62 \mathrm{mg}$ S-ibuprofen and $2.61 \mathrm{mg}$ RS-ibuprofen (so the actual value is $98.7 \%$ ee). Therefore, the estimated value by MagLev showed a $+0.1 \%$ difference. All the other 5 runs also showed errors $<1.0 \%$. These results suggest that MagLev can be used as a simple and efficient method for analysis of enantiomeric purity and enantioenrichment from a mixture containing the enantiomer and racemate.

Table 3. Enantiomeric purity of S-ibuprofen (top population) after four sequential density-separations using MagLev.

\begin{tabular}{c|cccc}
\hline $\begin{array}{c}\text { Number of } \\
\text { Separations }\end{array}$ & 1 & 2 & 3 & 4 \\
\hline $\begin{array}{c}\text { Purity of top } \\
\text { population }\end{array}$ & $95.1 \%$ ee & $97.2 \%$ ee & $98.6 \%$ ee & $99.2 \%$ ee \\
\hline
\end{tabular}

Beginning with an initial mixture having enantiomeric purity of $\sim 50 \%$ ee, we obtained $\sim 5 \%$ wt of the racemic compound "impurity" crystals in the top population after separation in Maglev (run 1 in Table 1). This result inspired our interest to perform iterative purification steps to increase \%ee. We removed the top population of crystals and dispersed them in a fresh paramagnetic solution with the aid of sonication and ran through another density-separation in MagLev (with the same operating procedure, $2^{\text {nd }}$ separation). The purity of the top population increased from $95.1 \%$ ee to $97.2 \%$ ee after the $2^{\text {nd }}$ separation. Then we carried out the same separation 
procedure again on the top population ( $3^{\text {rd }}$ separation). By repeating the separation procedure for a total of four times, we obtained $99.2 \%$ ee purity in the top population. We include a figure in the supporting information illustrating the procedure for iterative separations of the top population and table 3 summarizes the enantiomeric purity of the top population after each separation step. As the number of separations increased, the enantiomeric purity of the top population increased. After four sequential separations, an initial mixture of $\sim 50 \%$ ee was purified to a $99.2 \%$ ee product. The yield (defined as the percentage of final product mass over the initial S-ibuprofen mass) was $99.5 \%$. These results indicate that the "impurity" crystals can be gradually removed from a population of crystals by multiple-stage separations using MagLev. The improvement in separation with the use of ultrasonication between successive separations indicates that some type of agglomeration or physical trapping of "impurity" crystals occurs. In addition, it appears that small particles play an important role in the "impurity" particles since (1) good separation was observed during separation of two millimeter sized crystals; (2) crystals smaller than $2 \mu \mathrm{m}$ are strongly affected by Brownian motion and thus difficult to separate into a cluster by MagLev.

\section{Conclusions}

We have demonstrated that MagLev can be used as a simple and efficient method for analyzing enantiomeric purity and chiral separation in pharmaceutical industry. We summarize some highlights here as follows: (1) Typically, separating a racemic compound and its corresponding enantiomers requires significant work to develop a HPLC method or understand complex phase diagrams regarding different solvents and temperatures. In using MagLev method, none of the above is a concern. A small density difference $\left(0.01 \sim 0.02 \mathrm{~g} / \mathrm{cm}^{3}\right)$ that almost exists in every chiral system is good enough for separation. (2) This method can be used for continuous separation as well. Winkleman et al. used a microfluidic device combined with magnets to continuously separate and collect polystyrene particles of different densities in a flowing stream of aqueous $\mathrm{GdCl}_{3}$ solution. ${ }^{16,17}$ Although the diameters of these polystyrene particles $(75 \sim 150 \mu \mathrm{m})$ are larger than the crystals used here, our experiments here showed that $\sim 80 \%$ of particles (volumetric percentage by visual inspection) reached their equilibrium positions within 5 minutes while it takes $\sim 12 \mathrm{~h}$ for the other $\sim 20 \%$ of particles to separate. We therefore believe that it is reasonable to achieve a high degree of separation in a continuous system as long as some considerations are taken into the process design regarding particle size and separation time. (3) It works well with small quantities which can be hard for differential crystallization method. In pharmaceutical drug discovery phase, thousands of new compounds are produced with only a few milligrams for screening. With MagLev, highly pure enantiomer compounds can be easily obtained for further tests. (4) Because the amount of separated racemic and enantiopure crystals are visible, MagLev provides an instrument-free method of detecting small quantities of racemic crystals (or any other impurities of different density) in a bulk of enantiopure crystals. (5) This method provides both highly pure racemic and enantiopure crystals for future use. (6) It gives a high degree of separation in a single step (from $\sim 50 \%$ ee to $\sim 95 \%$ ee).

In summary, the use of MagLev provides a method that is lowcost, portable, non-destructive, and does not require electricity or extensive skill to operate. The analysis of enantiomeric purity was applied to mixtures of up to $98.7 \%$ ee, and the difference between the estimated values from MagLev and the actual values are all $<1.0 \%$. By applying four sequential separations using MagLev, we obtained a highly pure S-ibuprofen product in $99.2 \%$ ee starting from a $50 \%$ ee mixture with the racemate. In addition, this method developed for chiral analysis and separation is not limited to the pharmaceutical industry, but may also be used as a general tool for analysis and purification in any chiral system that crystallizes into forms that differentiate between chiral and racemic products. The impurities are not limited to racemic compound crystals but can apply to any compounds with different densities than that of the pure enantiomer. We believe developing a continuous separation system using MagLev has potential applications in purifying enantiomers from products of asymmetric synthesis reactions in the production of chiral drugs. It could be an alternative method to solution-based separations (e.g., HPLC or formation of diastereomers), especially in resource-limited settings where these methods are inaccessible or expensive to develop.

\section{Notes and references}

a Department of Chemical Engineering, Massachusetts Institute of Technology. 77 Massachusetts Avenue, Cambridge, MA 02139 (USA). E-mail: myerson@mit.edu

${ }^{b}$ Department of Chemistry and Chemical Biology, Harvard University. 12 Oxford Street, Cambridge, MA 02138 (USA). E-mail: gwhitesides@gmwgroup.harvard.edu

Electronic Supplementary Information (ESI) available: Experimental setup, procedures, materials, DSC analysis, more examples and photos. See DOI: $10.1039 / \mathrm{c} 000000 \mathrm{x} /$

1 N. M. Maier, P. Franco, W. Lindner, J. Chromatogr.A., 2001, 906, 3. 2 A. M. Thayer, $C \& E N ., 2007, \mathbf{8 5}, 11$.

3 R. J. D'Amato, M. S. Loughnan, E. Flynn, J. Folkman, Proc. Natl. Acad. Sci., 1994, 91, 4082.

4 I. K. Reddy, R. Mehvar (2004). Chirality in drug design and development. CRC Press.

5 S. S. Adams, P. Bresloff, \& C. G. Mason, J. Pharm. Pharmacol., 1976, 28, 256.

6 H. Federsel, J. Nat. Rev. Drug Discovery, 2005, 4, 685.

7 G. Gübitz, M. G. Schmid, Biopharm. Drug Dispos., 2001, 22, 291.

8 V. Dupray (2012), Recrystallization of Enantiomers from Conglomerates, Recrystallization, Prof. Krzysztof Sztwiertnia (Ed.), ISBN: 978-953-51-0122-2, InTech, DOI: 10.5772/33746.

9 H. Lorenz, F. Capla, D. Polenske, M. P. Elsner, A. SeidelMorgenstern, J. Univ. of Chem. Technol. and Metall., 2007, 42, 5.

10 K. A. Mirica, S. S. Shevkoplyas, S. T. Phillips, M. Gupta, G. M. Whitesides, J. Am. Chem.Soc., 2009, 131, 10049.

11 A. Winkleman, R. Perez-Castillejos, K. L. Gudiksen, S. T. Phillips, M. Prentiss, G. M. Whitesides, Analytical chemistry, 2007, 79, 6542.

12 M. R. Lockett, K. A. Mirica, C. R. Mace, R. D. Blackledge, G. M. Whitesides, J.Forensic Sci., 2013, 58, 40.

13 M. B. J. Atkinson et al. Angew. Chem.Int. Ed.. 2013, 52, 10208.

14 A. M. Evans, Clin. Rheumatology, 2001, 20, S9.

15 I. B. Rietveld, M. Barrio, B. Do and J. L. Tamarit, R. Céolin, J.Phys. Chem. B, 2012, 116, 5568 .

16 A. Winkleman, R. Perez-Castillejos, K. L. Gudiksen, S. T. Phillips, M. Prentiss and G. M. Whitesides, Anal. Chem., 2007, 79, 6542.

17 K. A. Mirica, S. T. Phillips, S. S. Shevkoplyas, and G. M. Whitesides, J. Am. Chem. Soc. 2008, 130, 17678. 